\title{
AVALIAÇÃO DE DESEMPENHO ORGANIZACIONAL NO SETOR COMERCIAL DE UMA EMPRESA DE CURSOS PROFISSIONALIZANTES
}

\section{EVALUATION OF ORGANIZATIONAL PERFORMANCE IN THE COMMERCIAL SECTOR OF A PROFESSIONALIZING COURSE COMPANY}

\author{
Cláudio Bezerra Leopoldino* \\ Jessé da Silva Oliveira**
}

\begin{abstract}
RESUMO
O presente artigo aborda uma pesquisa realizada em uma instituição de ensino profissionalizante, a respeito dos processos de avaliação de desempenho individual dos colaboradores do seu setor comercial. O objetivo é descrever o processo vigente e propor um processo alternativo aprimorado, com base nas limitações identificadas. A investigação foi de natureza qualitativa e empregou a observação, o questionário e a análise de documentos como meios de coleta de dados. Observou-se que a empresa era inflexível em seus processos avaliativos, demitindo sumariamente os empregados que não atingissem as metas em curto espaço de tempo, gerando rotatividade e custos. Adicionalmente, certos aspectos básicos da avaliação de desempenho como o feedback e transparência eram ignorados. Constatou-se que com pequenas modificações, o modelo vigente poderia, sem perder suas características, equilibrar melhor a avaliação do desempenho dos indivíduos e o desenvolvimento das pessoas que compõem o corpo funcional da organização.
\end{abstract}

Palavras-chave: Avaliação de Desempenho Individual; Análise de Processos; Recursos Humanos.

\begin{abstract}
This article addresses a survey carried out in a vocational education institution regarding the individual performance appraisal processes of employees in its commercial sector. The objective is to describe the current process and propose an improved alternative process, based on the identified limitations. The research was qualitative in nature and employed observation, questionnaire and document analysis as means of data collection. It was observed that the company was inflexible in its evaluation processes, summarily dismissing employees who did not reach their goals in a short period of time, generating turnover and costs. Additionally, certain basic aspects of performance appraisal such as feedback and transparency were ignored. It was found that with minor modifications, the current model could, without losing its characteristics, better balance the evaluation of the individual performance and the development of the people who make up the body of the organization.
\end{abstract}

Keywords: Performance Appraisal; Process analysis; Human Resources.

Data de submissão: 19 de dezembro de 2020

\footnotetext{
*UFC — Universidade Federal do Ceará. E-mail: claudio.leopoldino@ufc.br

** UFC — Universidade Federal do Ceará. E-mail: jesse.s.o@ hotmail.com

Revista Eletrônica do Alto Vale do Itajaí - REAVI, v.10, nº 16, p. 034-045, ago. 2021.
} 
Data de aprovação: 27 de abril de 2021

Disponibilidade: DOI 10.5965/2316419010152021034

\section{INTRODUÇÃO}

A avaliação sistemática de desempenho dos recursos humanos permite melhoria da ação do quadro de pessoal e insights a respeito dos processos da organização. Nesse contexto, tornam-se relevantes, a prática e o estudo contínuo da avaliação de desempenho individual, pois através dela é possível obter resultados para a organização, utilizando-se de diversos métodos (AGGARWAL; THAKUR, 2013; CAMPOS; DOS SANTOS; RODRIGUES, 2014; KHANNA; SHARMA, 2014; MARRAS, 2012). A prática da avaliação de desempenho dos colaboradores está associada a melhorias nos processos, na tomada de decisões, maior satisfação e comprometimento organizacional e, por fim, a uma melhor performance organizacional (AYERS, 2015; BORTOLUZZI; ENSSLIN; ENSSLIN, 2015; MARTINS; ENSSLIN; DUTRA, 2018; ISLAMI; MULOLLI; MUSTAFA, 2018; PINHO et al., 2020).

Acredita-se que o investimento nos colaboradores culmine no desenvolvimento e aperfeiçoamento das atividades realizadas por eles. Neste sentido, a avaliação de desempenho poderia ser empregada para direcionar promoções e outras recompensas, promover feedback, aumentar a satisfação, a motivação e a eficiência dos empregados (AYERS, 2015; DENISI; MURPHY, 2017; KHANNA; SHARMA, 2014), podendo ainda facilitar a implementação das estratégias organizacionais (MARTINS; ENSSLIN; DUTRA, 2018).

Entretanto, os benefícios prometidos podem não ser sempre entregues. Caso a avaliação de desempenho não siga os procedimentos adequados, não disponha de recursos ou apoio suficiente para tornar-se efetiva, não surtirá o efeito buscado e poderá até mesmo apresentar-se danosa à organização e seus integrantes (AYERS, 2015; KHANNA; SHARMA, 2014; REIFSCHNEIDE, 2008; SACHS, 1995; SIQUEIRA, 2002). Dessa forma, a literatura aponta casos em que há descontentamento tanto de supervisores quanto dos empregados avaliados (KIM; HOLZER, 2016; KLEIN et al., 2019), tornando-se evidente a importância de se pesquisar organizações com práticas disfuncionais de avaliação de desempenho.

Tendo em consideração a presente problemática, este artigo tem como objetivo geral analisar os processos de avaliação de desempenho de recursos humanos, tendo como caso em estudo o setor comercial de uma instituição de ensino profissionalizante. Para tanto, os objetivos específicos são: descrever os processos de avaliação de desempenho vigentes; identificar os principais problemas enfrentados decorrentes de incorreções na avaliação de desempenho; e, por fim, propor um modelo aprimorado, com base na literatura sobre o tema e nas limitações identificadas.

Assim, espera-se que este estudo possa contribuir com as demais pesquisas desenvolvidas no campo da avaliação de desempenho, ao focar nas práticas disfuncionais deste processo em um estudo de caso, gerando ideias de alternativas em termos de melhorias e modelos que estejam mais próximos dos diversos contextos organizacionais.

Revista Eletrônica do Alto Vale do Itajaí - REAVI, v.10, nº 16, p. 034-045, ago. 2021. 


\section{AVALIAÇÃO DE DESEMPENHO NAS ORGANIZAÇÕES}

A atuação dos profissionais em uma organização é fonte de diferenciais competitivos e de eficiência, mas pode levar à baixa produtividade e a erros, quando mal gerenciada (ISLAMI; MULOLLI; MUSTAFA, 2018). Neste contexto, mensurar o desempenho do pessoal envolvido é uma forma de obter informações para aprimorar a qualidade da gestão. A avaliação de desempenho realiza análises a respeito da diferença ou defasagem entre o que foi exigido e o que foi entregue, concentrando o foco da averiguação nesse intervalo entre o processo ideal e o real. A avaliação de desempenho permite diferenciar o insucesso, o desempenho medíocre e a performance desejada dos colaboradores de uma organização (REIFSCHNEIDE, 2008). Essa prática de gestão de recursos humanos pode ser observada em grandes e pequenas empresas e em instituições públicas e privadas, além de organizações militares (KHANNA; SHARMA, 2014; ODELIUS; DOS SANTOS, 2008), e já é pesquisada há pelo menos um século (DENISI; MURPHY, 2017).

Marras (2012) afirma que a avaliação de desempenho possui três focos principais, a aferição do potencial; o desenvolvimento profissional; e a realização de metas e objetivos. A partir de cada enfoque, é possível utilizar diversos formalismos para a realização da avaliação, entre eles: os métodos tradicionais, que utilizam a escala gráfica, incidentes críticos e escolha forçada; e os métodos modernos, utilizando a avaliação de desempenho por objetivos, autoavaliação e avaliação 360 (AGGARWAL; THAKUR, 2013; ISLAMI; MULOLLI; MUSTAFA, 2018; KHANNA; SHARMA, 2014). Adicionalmente, outros métodos, como a análise multicritério e a avaliação por competências, podem ser utilizados (BORTOLUZZI; ENSSLIN; ENSSLIN, 2015; CAMPOS; DOS SANTOS; RODRIGUES, 2014), além de métodos mistos. A conjugação de mais de uma técnica apresenta potencial para trazer resultados relevantes, permitindo ângulos diferentes de visão, além de poder impactar positivamente a satisfação e a motivação dos colaboradores (IDOWU, 2017).

Entretanto, apesar de as organizações poderem se beneficiar com a avaliação de desempenho de seus integrantes de diversas formas, algumas delas ainda não aproveitam de forma efetiva os impactos positivos desse procedimento (AYERS, 2015; KLEIN et al., 2019; SACHS, 1995). As causas de insucesso podem ser várias, tais como a falta de recursos, a ausência de apoio da gerência superior, a ausência de alinhamento estratégico ou erros de implantação.

Tampouco se pode afirmar que a avaliação de desempenho individual é unanimemente aprovada. Seus críticos consideram esse processo desnecessário, prolixo e causador de desmotivação para os funcionários, devido à falsa impressão de que ela serve apenas para criticar o resultado insatisfatório de algum colaborador (SIQUEIRA, 2002). Outra crítica é a de que a avaliação de desempenho individual carece de ideias recentes que a ampliem e de maior interação com teorias de outras áreas de conhecimento (MARTINS; ENSSLIN; DUTRA, 2018). Khanna e Sharma (2014) registraram que há diversos relatos de descontentamento quanto à avaliação de desempenho individual, posição corroborada por Kim e Holzer (2016).

Reifschneide (2008) afirma que são frequentes as avaliações de desempenho que assumem o caráter de julgamentos arbitrários a partir de critérios equivocados, comunicados post factum aos empregados, e originados da falta de preparo e infraestrutura. Convém,

Revista Eletrônica do Alto Vale do Itajaí - REAVI, v.10, nº 16, p. 034-045, ago. 2021. 
portanto, utilizar com prudência e consciência os métodos, processos e conceitos da avaliação de desempenho, para evitar resultados contrários aos desejados.

A adoção de boas práticas nos processos de avaliação individual torna mais fácil a obtenção de resultados mais efetivos. Destacam-se entre as recomendações da literatura, ações como a avaliação do potencial dos empregados, o estímulo ao desenvolvimento das competências dos trabalhadores, o feedback a respeito do trabalho que está sendo realizado e a documentação do desempenho individual ao longo do tempo (AYERS, 2015; CAMPOS; DOS SANTOS; RODRIGUES, 2014; DENISI; MURPHY, 2017; ELLIOTT, 2015; IDOWU, 2017; KHANNA; SHARMA, 2014; MARRAS, 2012). A informatização do processo traz maior escala, agilidade, organização e acesso mais rápido dos avaliados a informações sobre o seu próprio desempenho, afetando positivamente em sua performance individual (SHARMA; SHARMA, 2017).

\section{METODOLOGIA}

Quanto aos procedimentos metodológicos, optou-se pela realização de uma pesquisa aplicada, tendo como campo de estudo o setor comercial de uma instituição de ensino profissionalizante. Em relação à natureza da pesquisa, é caracterizada como descritiva (RAMOS, 2009). No que concerne à abordagem da pesquisa, classifica-se como qualitativa, com escolha da organização investigada por conveniência (GIL, 2008).

Quanto aos instrumentos de coleta, foram utilizados a observação, o questionário e a análise de documentos. A observação foi escolhida pela vantagem de não necessitar de intermediações, sendo possível colher e analisar as informações de forma clara e direcionada, sendo classificada como não estruturada, pois havia liberdade na forma de observar (GIL, 2008).

O questionário foi utilizado devido à possibilidade de reunir informações primárias, traduzir os objetivos da pesquisa através de perguntas direcionadas, possuir menor custo e permitir o anonimato dos respondentes, os quais foram considerados do tipo natural por fazerem parte da própria organização (RAMOS, 2009). Responderam o questionário tanto o supervisor como seus subordinados alocados em um dos centros de venda da rede de cursos profissionalizantes. O instrumento foi composto por nove questões direcionadas para os consultores e oito questões direcionadas para o supervisor.

As perguntas foram mescladas em abertas e fechadas e as respostas dos consultores foram apresentadas anonimamente. Os questionários abordaram tópicos da avaliação de desempenho organizacional, identificando a prática de algum processo de avaliação, quais os fatores eram avaliados no processo e quais as expectativas eram geradas com o processo vigente. A coleta foi realizada na última semana de maio de 2018.

Por fim, os principais documentos analisados foram os relatórios gerados pelo sistema de avaliação da própria empresa nos períodos de avaliação, os quais descreviam os resultados de cada colaborador no que se refere ao número de matrículas vendidas, de cancelamentos efetuados e da taxa de conversão alcançada. Salienta-se que não foi autorizado pela empresa expor informações acerca do número de vendas atingido ou exigido pelo setor. Tais informações foram, portanto, descartadas das análises.

Revista Eletrônica do Alto Vale do Itajaí - REAVI, v.10, nº 16, p. 034-045, ago. 2021. 
Desse modo, através da análise documental, dos resultados gerados pela observação e dos questionários, foram obtidas informações trianguladas acerca do modelo de avaliação vigente, compreendendo qual a visão do líder e dos liderados a respeito da avaliação de desempenho realizada no setor. $\mathrm{O}$ modelo vigente foi validado pela empresa, sendo utilizado o referencial teórico desta pesquisa para fornecer subsídios para a formulação de proposta de um modelo aprimorado, que se supera as limitações identificadas no modelo atual, mantendo as características no modelo vigente que eram valorizadas pela organização.

\section{DISCUSSÃO DO CASO}

A empresa investigada é uma rede de escolas de cursos profissionalizantes na área da educação em internet e informática. Possui mais de 45 escolas em todo o país, operando desde 1984, com matriz na cidade de Curitiba. Os cursos são destinados a jovens de 11 a 20 anos, tendo como público-alvo as classes $\mathrm{C}$ e $\mathrm{D}$, para o qual a empresa oferece uma grade completa de módulos, que vão desde conceitos básicos da computação até manutenção e configuração de computadores, oferecendo assim, qualificação para jovens que buscam oportunidades no mercado de trabalho.

A organização concentra seus esforços nos setores pedagógico, telemarketing, administrativo e comercial. Como destacado anteriormente, o foco deste artigo é a avaliação de desempenho organizacional no setor comercial, cuja função é alavancar as vendas na organização através da promoção das matrículas nos cursos profissionalizantes.

A empresa investigada possui em sua cultura organizacional a premissa de que o colaborador do setor comercial deve atingir mensalmente a meta mínima estabelecida. Caso essa meta não seja alcançada no mês vigente, o colaborador deverá atingi-la no mês seguinte. Caso contrário, ocorrerá o desligamento da empresa.

As constantes demissões geram considerável rotatividade no setor, tendo como consequência a permanente necessidade de recrutar pessoal, gerando custos para a empresa, além de abalar o comprometimento dos colaboradores remanescentes.

\subsection{MODELO VIGENTE}

A empresa estudada utiliza traços do método de avaliação mista, apresentando elementos de auto avaliação, na qual o trabalhador avalia o próprio desempenho, e da avaliação por resultados, uma vez que é analisada a defasagem entre o resultado esperado e o atingido, identificando necessidades de intervenções (AGGARWAL; THAKUR, 2013; KHANNA; SHARMA, 2014). A avaliação é realizada no início de cada mês, com cada consultor. Vale ressaltar que não há um momento definido para prática do feedback individual, embora seja comum pelo menos uma vez por semana o supervisor comentar a respeito do resultado e de possíveis dificuldades que possam existir com cada consultor.

O fluxo principal no modelo vigente de avaliação foi representado graficamente por um diagrama de processos, sendo composto por cinco atividades: a divulgação dos resultados das vendas; a análise dos resultados; a conferência do alcance da meta mínima de vendas com detalhamentos; a identificação das dificuldades enfrentadas e o desenvolvimento de projeções

Revista Eletrônica do Alto Vale do Itajaí - REAVI, v.10, nº 16, p. 034-045, ago. 2021. 
dos resultados para a próxima meta. A Figura 1 apresenta o modelo vigente de avaliação de desempenho utilizado no setor comercial, validado após a coleta de dados.

\section{Figura 1 - Avaliação de desempenho vigente}

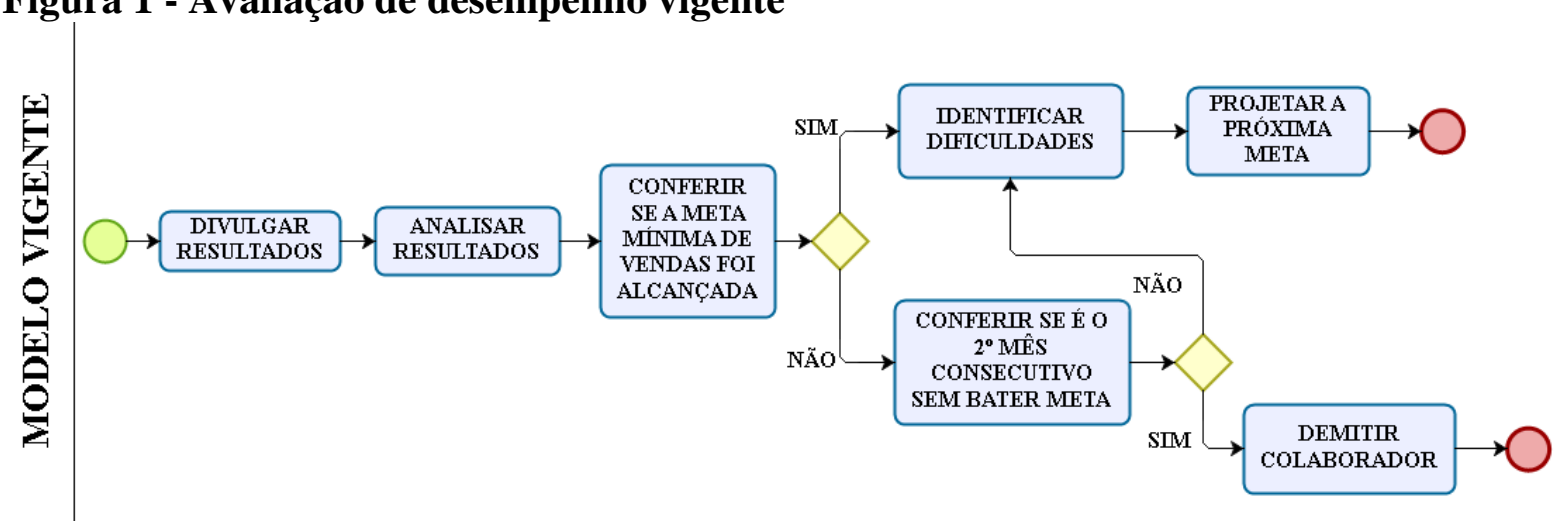

Fonte: Elaborada pelos autores com base nos dados da pesquisa.

No primeiro momento, ocorre a divulgação dos resultados alcançados em relação ao número de matrículas vendidas, cancelamentos realizados e taxas de conversão alcançadas. Tais informações são geradas através do sistema em forma de relatório. Salienta-se que o número de matrículas realizadas no período possui uma maior importância para a organização e é analisado com maior atenção.

A segunda atividade envolve a análise dos resultados do período em relação aos indicadores mencionados anteriormente. Cada fator possui previamente um limite a ser atingido, estabelecido pela supervisão: o número de cancelamentos deve estar dentro de uma porcentagem determinada e a taxa de conversão dentro do limite de atendimentos realizados e matrículas vendidas.

A terceira etapa do fluxo é formada por uma análise mais profunda a respeito do número de matrículas vendidas. Por ser um fator preponderante, torna-se oportuno mencionar que há uma atenção específica do supervisor para conferir se o avaliado atingiu a meta mínima. Quando o avaliado atinge a meta definida, a expectativa por parte da supervisão é de que o resultado seja mantido. Já quando o avaliado não atinge a meta mínima, é necessário conferir se já é o segundo mês consecutivo que o colaborador não atinge a meta determinada. Caso seja o segundo mês consecutivo sem alcançar a meta mínima, o colaborador será demitido, entretanto, caso tenha sido o primeiro mês que o colaborador não tenha alcançado a meta, ele terá mais uma oportunidade de alcançar pelo menos a meta mínima no período mensal seguinte.

Entretanto, foi percebido que há determinadas exceções para a demissão de algum colaborador que não tenha alcançado a meta por dois meses consecutivos. Tais exceções são determinadas exclusivamente pela liderança da organização, representada pelo supervisor e diretor da unidade.

Revista Eletrônica do Alto Vale do Itajaí - REAVI, v.10, nº 16, p. 034-045, ago. 2021. 
No quarto momento ocorre a identificação das dificuldades enfrentadas durante o período, comentando-se sobre as limitações em todo o processo. De acordo com o grau de complexidade, o problema é analisado de forma aprofundada, buscando soluções práticas. Em alguns casos, essas experiências são compartilhadas com toda a equipe.

No quinto momento é desenvolvida a projeção do resultado para o mês seguinte, na qual o avaliado estipula uma meta a ser alcançada perante seu supervisor. Cabe ressaltar que a meta mínima é o número que orienta o resultado estipulado a ser alcançado.

A avaliação de desempenho é finalizada após cada consultor ter esse diálogo com o supervisor para prestação de contas do resultado do mês. Ressalta-se que não existe um momento exclusivo de feedback definido no processo para desenvolver de forma estruturada a performance do colaborador. Inexiste portanto, no processo vigente, oportunidade para uma avaliação formativa dos empregados (ELLIOTT, 2015; REIFSCHNEIDE, 2008).

\subsubsection{Dificuldades e limitações do processo de avaliação}

Alguns aspectos foram observados no setor da empresa em estudo e se revelaram como restrições e obstáculos ao processo de avaliação. As principais restrições constatadas envolvem a falta de ferramenta informatizada, a falta de estruturação e formalização, a reduzida transparência aos avaliados, e a ausência de feedback e de planejamento de desenvolvimento individual.

A empresa não possui um software específico para a avaliação de desempenho, ou outra ferramenta informatizada que ofereça tal funcionalidade. $\mathrm{O}$ acompanhamento da evolução dos colaboradores se torna, em virtude disso, mais lento e superficial.

A empresa não normatizou ou estruturou o processo de avaliação de forma sistemática e nem possui questionários, planilhas ou formulários de avaliação formalizados, dependendo apenas do supervisor para divulgação e análise dos resultados. Como consequência o processo perde coesão e pode ser flexibilizado por gerentes complacentes, ou ser utilizado com excesso de rigor, por parte de gestores excessivamente rígidos.

Das informações geradas pela avaliação de desempenho individual, apenas o resultado da meta vigente é apresentado para o colaborador, não deixando claro se existem outras finalidades com o seu uso e restringindo a transparência aos avaliados.

Constatou-se ainda que não há um momento exclusivo para o feedback individual com os colaboradores e não há um planejamento de desenvolvimento individual claro para o colaborador. Dificulta-se desta forma o aperfeiçoamento dos membros da equipe de vendas e a correção de erros e omissões. Não foram observados protocolos ou direcionamentos para assumir promoções ou alocação em outros cargos.

Dessa forma, os itens listados anteriormente representam as dificuldades e limitações da organização. Tais problemas demonstram que a empresa pode desenvolver esse setor através de novas práticas e aperfeiçoamento da avaliação de desempenho.

\subsection{MODELO PROPOSTO}

O modelo proposto levou em conta as necessidades da empresa, que depende da alta performance de vendas, e ao mesmo tempo agregando práticas que mitigam as restrições

Revista Eletrônica do Alto Vale do Itajaí - REAVI, v.10, n 16, p. 034-045, ago. 2021. 
constatadas no modelo vigente. Em seu fluxo principal, o processo proposto possui sete atividades distintas: a divulgação dos resultados; a análise dos resultados; a conferência do alcance da meta com bifurcações caso o colaborador não tenha alcançado o resultado mínimo exigido; a identificação das dificuldades enfrentadas; o compartilhamento do feedback; a definição de ações a serem tomadas e o desenvolvimento de projeções para a próxima meta.

No primeiro momento ocorre a divulgação dos resultados alcançados em relação ao número de matrículas, cancelamentos realizados e taxas de conversão. Já a segunda atividade do fluxo principal compreende a análise dos resultados relacionados aos números de cancelamentos, taxas de conversão e matrículas. Cada item possui um limite já determinado e deve ser usado como direcionamento para os avaliados.

No terceiro momento, realiza-se a conferência detalhada do alcance da meta mínima das matrículas vendidas, existindo uma bifurcação para identificar se essa meta foi alcançada. Caso tenha sido alcançada, o avaliado segue com a avaliação no fluxo principal, caso não tenha sido alcançada será necessário identificar se ele já participou de alguma avaliação de desempenho anterior. Se o avaliado não tiver participado de nenhuma avaliação anterior, segue o fluxo principal, entretanto se já tiver participado será necessário identificar melhorias em seu resultado. Caso haja alguma melhoria entre uma avaliação e outra, deve-se seguir no fluxo principal, mesmo que a meta mínima de vendas de matrículas não tenha sido alcançada.

Uma vez que não seja identificada nenhuma melhoria perceptível, o avaliador deverá definir alguma ação a ser tomada. Nesse momento será definido se esse colaborador será demitido; participará de algum treinamento específico; participará da criação de um plano de desenvolvimento individual ou será alocado em outro cargo. Vale ressaltar que a decisão de demitir um colaborador devido ao não atingimento da meta mínima de vendas por dois meses consecutivos consiste na regra geral aplicada na organização, como já fora mencionado. Entretanto, a decisão de demitir o colaborador, nessa situação, continua dependendo de requisitos determinados pelo gerente e supervisor da unidade. Sugere-se, portanto, que sejam divulgados os critérios estabelecidos e que fique claro para os colaboradores quais os protocolos e direcionamentos relacionados à demissão.

No quarto momento há a identificação das limitações e dificuldades apresentadas pelo colaborador durante o período avaliado, as quais servirão de apoio para criação de ações específicas e estudos de caso a serem compartilhados com a equipe. Na quinta atividade ocorre o compartilhamento do feedback, no qual o supervisor comenta sobre os principais aspectos do trabalho desenvolvido pelo avaliado, considerando sempre a busca pelo aperfeiçoamento das atividades realizadas.

No sexto momento desenvolve-se a tomada de decisões baseada nas informações geradas, representada pela criação de planos de desenvolvimento individuais; promoções; treinamentos específicos; alocação em outros cargos e demissão.

Revista Eletrônica do Alto Vale do Itajaí - REAVI, v.10, n 16, p. 034-045, ago. 2021. 


\section{REAVI}

Figura 2 - Novo modelo de avaliação de desempenho para a empresa.

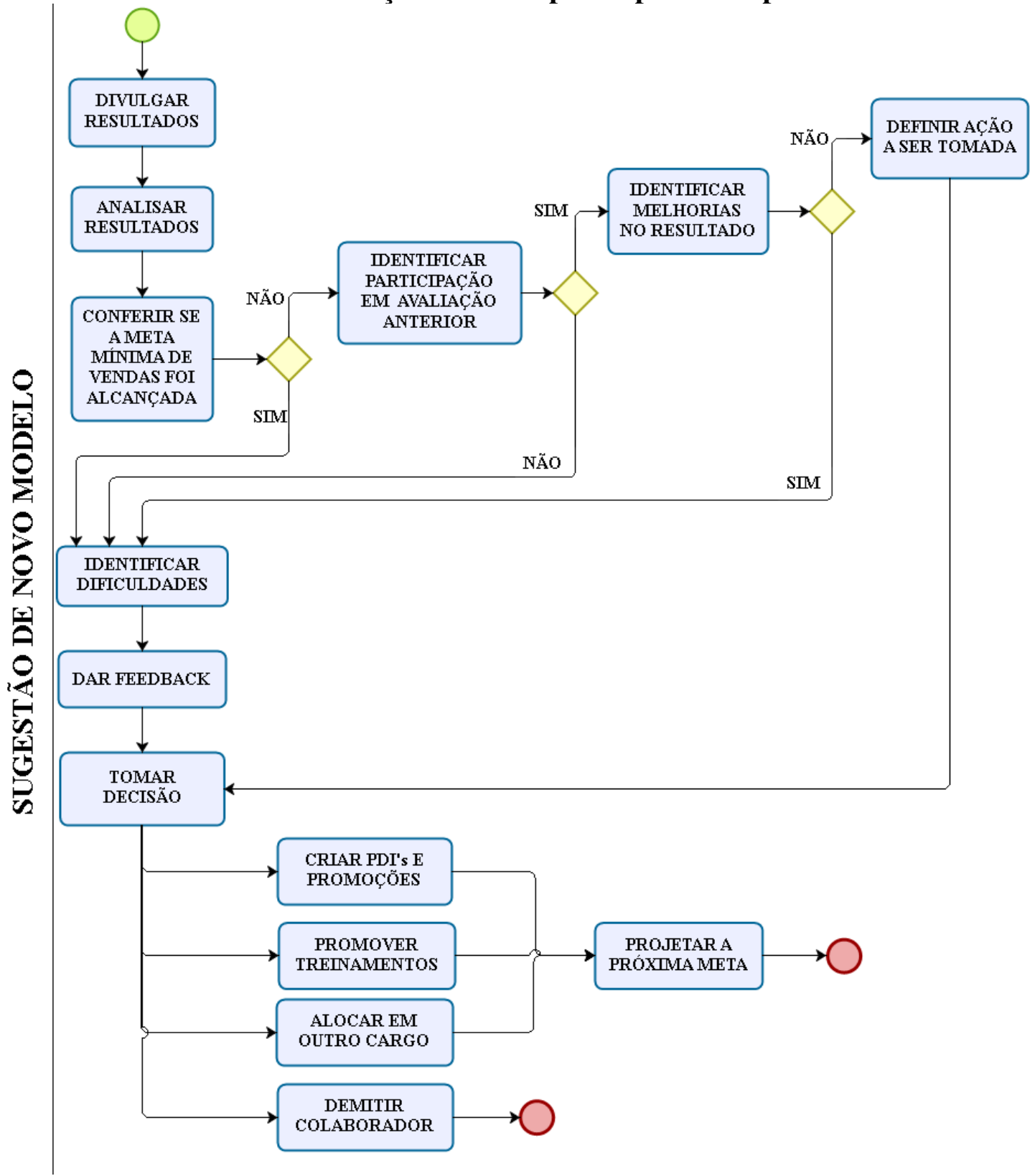

Fonte: Elaborada pelos autores com base nos dados da pesquisa.

No sétimo processo, criam-se projeções de resultados para a próxima meta. Os participantes, considerando as informações geradas e dificuldades enfrentadas no período, devem projetar a nova meta a ser alcançada, ressaltando que caso haja qualquer alteração nas metas, para cima ou para baixo, o crescimento deve ser gradual, evitando a proposta de objetivos fora da realidade. Vale acrescentar que todas as informações coletadas e produzidas devem ser registradas pelo supervisor para futuras tomadas de decisão, no próprio sistema da

Revista Eletrônica do Alto Vale do Itajaí - REAVI, v.10, nº 16, p. 034-045, ago. 2021. 
empresa ou através de alguma ferramenta online de fácil acesso. A Figura 2 apresenta a sequência de atividades para o modelo de avaliação de desempenho proposto.

$\mathrm{O}$ novo fluxo principal aprimorou o processo vigente, mantendo o atendimento das necessidades de controle apresentadas pela empresa. Salienta-se que houve três mudanças processuais consideráveis em relação ao modelo vigente.

A primeira mudança foi a criação de bifurcações para identificar o alcance da meta mínima, a participação em avaliação anterior e a identificação de melhorias no resultado. Com essa mudança, no caso do consultor que não tenha alcançado a meta mínima, mas tenha obtido melhorias em seu resultado, a avaliação deve prosseguir no fluxo principal.

A segunda modificação consistiu na criação de um momento exclusivo de feedback entre supervisor e avaliado, para discussões a respeito do desenvolvimento de cada colaborador, considerando momentos de dificuldade e crescimento do avaliado.

Por fim, a última mudança foi a criação e seleção de ações a serem tomadas a partir dos resultados obtidos, como ir direto para projeção da próxima meta; criação de promoções e planos de desenvolvimento individuais; criação de treinamentos; alocações em outros cargos e demissão. Manteve-se o ciclo mensal de avaliação, considerado um elemento positivo do modelo vigente.

Em suma, o Quadro 1 destaca as principais características do novo modelo, em comparação ao anterior.

\section{Quadro 1 - Resumo das características dos modelos de avaliação da empresa}

\begin{tabular}{|c|c|c|}
\hline Modelo Vigente & Restrições do Modelo Vigente & $\begin{array}{l}\text { Características } \\
\text { Proposto }\end{array}$ \\
\hline $\begin{array}{l}\text { - Empregados são avaliados com } \\
\text { base nas metas de vendas; } \\
\text { - Os colaboradores são demitidos } \\
\text { sumariamente se não atingirem a } \\
\text { meta de vendas por dois meses } \\
\text { seguidos; } \\
\text { - Fluxo principal composto por } \\
\text { cinco atividades: divulgação dos } \\
\text { resultados das vendas; análise; } \\
\text { conferência do alcance da meta } \\
\text { mínima de vendas; identificação } \\
\text { das dificuldades enfrentadas e } \\
\text { desenvolvimento de projeções } \\
\text { dos resultados para a próxima } \\
\text { meta. }\end{array}$ & $\begin{array}{l}\text { - Falta de software para auxiliar o } \\
\text { processo; } \\
\text { - Processo sem estruturação ou } \\
\text { formalização; } \\
\text { - Transparência insuficiente aos } \\
\text { avaliados; } \\
\text { - Ausência de feedback; } \\
\text { - Falta de planejamento de } \\
\text { desenvolvimento individual. }\end{array}$ & $\begin{array}{l}\text { - O processo proposto possui sete } \\
\text { atividades distintas para o fluxo } \\
\text { principal: divulgação dos } \\
\text { resultados; análise dos resultados; } \\
\text { conferência do alcance; } \\
\text { identificação das dificuldades; } \\
\text { feedback; definição de ações a } \\
\text { serem tomadas e o } \\
\text { desenvolvimento de projeções } \\
\text { para a próxima meta; } \\
\text { - Processo mais estruturado e } \\
\text { formalizado, com maior } \\
\text { transparência e feedback, } \\
\text { apresentando meios para o } \\
\text { aprimoramento } \\
\text { desenvolvimento individual em } \\
\text { contraposição às demissões } \\
\text { sumárias. }\end{array}$ \\
\hline
\end{tabular}

Fonte: Elaborado pelos autores.

As principais melhorias no processo proposto compreendem: representação gráfica e textual dos fluxos de atividades de avaliação de desempenho; padronização do modelo e registro das informações geradas para tomadas de decisão; criação de um momento de feedback

Revista Eletrônica do Alto Vale do Itajaí - REAVI, v.10, nº 16, p. 034-045, ago. 2021. 
entre o supervisor e consultor para desenvolvimento do avaliado e, por fim, a sugestão de ações a serem tomadas mediante as informações geradas com a avaliação, entre elas a demissão e criação de treinamentos. Cabe ressaltar que, relacionada à limitação do software, sugere-se a compra de algum programa específico de avaliação de desempenho individual que facilite e dinamize esse processo, trazendo benefícios para a organização e para os colaboradores avaliados (SHARMA; SHARMA, 2017). Buscou-se, com a proposição do modelo, um maior equilíbrio entre a gestão do desempenho individual e o desenvolvimento das pessoas que compõem a organização (ELLIOTT, 2015). A realidade, entretanto, não apresenta no momento perspectiva de mudança na empresa investigada, a qual está ciente do modelo proposto, mas não realizou nenhum investimento no sentido de colocá-lo em prática.

\section{CONCLUSÃO}

O presente texto caracteriza-se como reflexão sobre a prática avaliativa, mostrando a situação de uma empresa em que demissões sumárias geravam rotatividade e custos e na qual certos aspectos básicos da avaliação de desempenho como o feedback e transparência eram ignorados. Constatou-se que com pequenas modificações, o modelo vigente poderia, sem perder suas características, equilibrar melhor a avaliação do desempenho e o desenvolvimento das pessoas que compõem o corpo funcional da organização.

Como principais limitações da pesquisa podem ser elencadas a falta de acesso a informações da matriz e restrições de divulgação dos dados obtidos junto à filial onde ocorreram as entrevistas e a coleta de dados. Adicionalmente, pode ser citado como restrição o fato de utilizar amostragem por conveniência e o horizonte limitado de tempo, com recorte transversal.

Diversas são as possibilidades de investigações futuras, tais como estudos setoriais e multicasos, e o acompanhamento de processos de avaliação de desempenho de recursos humanos ao longo do tempo. Acredita-se que avaliação de desempenho individual, realizada de forma fundamentada, apresenta potencial para melhorar a performance dos colaboradores e ao mesmo tempo trazer benefícios financeiros e não financeiros para a organização.

\section{REFERÊNCIAS}

AGGARWAL, A.; THAKUR, G. S. M. Techniques of performance appraisal-a review. International Journal of Engineering and Advanced Technology (IJEAT), v. 2, n. 3, p. 617-621, 2013.

AYERS, R. S. Aligning individual and organizational performance: Goal alignment in federal government agency performance appraisal programs. Public Personnel Management, v. 44, n. 2, p. 169-191, 2015.

BORTOLUZZI, S. C.; ENSSLIN, S. R.; ENSSLIN, L. Gestão estratégica de recursos humanos por meio de uma ferramenta de avaliação de desempenho organizacional. REVISTA PRODUÇÃO E ENGENHARIA, v. 5, n. 1, p. 443-461, 2015.

Revista Eletrônica do Alto Vale do Itajaí - REAVI, v.10, nº 16, p. 034-045, ago. 2021. 
CAMPOS, A. L.; DOS SANTOS, N. M. B. F.; RODRIGUES, J. L. K. Gestão de Recursos Humanos e Avaliação de Desempenho por Competências: análise do processo em uma empresa do Vale do Paraíba. Revista ENIAC Pesquisa, v. 3, n. 1, p. 33-53, 2014.

DENISI, A. S.; MURPHY, K. R. Performance appraisal and performance management: 100 years of progress?. Journal of Applied Psychology, v. 102, n. 3, p. 421, 2017.

ELLIOTT, K. Teacher Performance Appraisal: More about Performance or Development?. Australian Journal of teacher education, v. 40, n. 9, p. n9, 2015.

DALLABONA, L. F.; BEUREN, I. M. Relação da folga organizacional com medidas de desempenho de empresas brasileiras. In. CONGRESSO BRASILEIRO DE CUSTOS, 19., 2012. Rio Grande do Sul. Anais... Rio Grande do Sul, 2012. CD-ROM.

DALLABONA, L. F.; DIEL, F. J.; LAVARDA, C. E. F. Variabilidade de folga organizacional de empresas listadas nos níveis diferenciados de governança corporativa da BM\&FBovespa. Registro Contábil, v. 5, n. 2, p. 67-86, 2014.

GOSSELIN, M. The effect of strategy and organizational structure on the adoption and implementation of activity-based costing. Accounting, Organizations and Society. v. 22, n. 2, p. 105-122, 1997.

DEMO, P. Metodologia do conhecimento científico. São Paulo: Atlas.

Revista Eletrônica do Alto Vale do Itajaí - REAVI, v.10, n 16, p. 034-045, ago. 2021. 